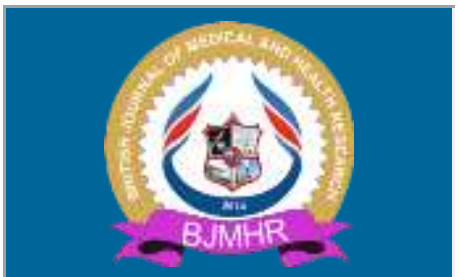

\title{
BJMHR
}

British Journal of Medical and Health Research Journal home page: www.bjmhr.com

\section{Preoperative Acetate Templating In Total Knee Arthroplasty: Does It Matter?}

\author{
Khairul NS*, Zulkifly AH. \\ Department of Orthopaedics, Traumatology and Rehabilitation, Kulliyyah of Medicine, \\ International Islamic University of Malaysia, Malaysia.
}

\begin{abstract}
Preoperative templating is a crucial step in total knee arthroplasty (TKA). This study was aimed to assess the accuracy of manual templating is reliable with the size for total TKA. Preoperative was performed on 100 patients prior to TKA. The acetate templating $\left(\right.$ Nexgen $^{\circledR}$, Zimmer $^{\circledR}, 20 \%$ magnification) was used in this study. Magnification of radiographs was measured according to standard protocol. The mismatch of standard templated sizes preoperatively and sizes implanted were observed and recorded. The accuracy and reliability of preoperative templating were assessed. Data were analysed using Student's t-test and ANOVA. Statistical analysis shows that there is no significant difference in the accuracy of templating and actual implant in both tibia and femur components $(P>0.05)$. The accuracy of template tibia component demonstrated $48 \%$ of accuracy and $95 \%$ within one size of mismatch. Meanwhile, in femoral, the component templating shown $47 \%$ accuracy and $97 \%$ within one size mismatch. There was no size mismatch for more than 2 sizes was noted. The study has shown that a higher tendency to template smaller size for both components in total knee arthroplasty is recommended. We concluded that the manual templating is an indispensable preoperative technique for TKA operations.
\end{abstract}

Keywords: Arthroplasty, Total knee arthroplasty, templating, preoperative 


\section{INTRODUCTION}

Preoperative planning of total knee arthroplasty is an essential part of the surgical procedure. The surgeon pursuits for optimal fit of the knee implant size and positioning in order to achieve the best reconstruction of the lower limb, to restore leg length and the position of the centre of rotation in the procedure. As a secondary institution for knee arthroplasty without access to digital templating software, this topic is quite significant to be conducted. The radiological image of Antero-Posterior (AP) and Medio-Lateral (ML) of the tibia and femoral components are crucial in deciding the implant size to be used $\left({ }^{1}\right)$. The accuracy and reliability of manual templating can be as references to surgeons to prepare the adequate implant sizes preoperatively for total knee arthroplasty (TKA). Preoperative planning enables surgeons to think in three-dimensional and thoughtful ways to improve surgery accuracy, shorten surgery time, and reduce complications. $\left({ }^{2-4}\right)$.

Preoperative radiograph templating in TKA is mandatory for estimation of implant size as it is an essential part of surgical procedures. This procedure is necessary for the surgeons not only to select the implant size but also to correct the alignment, orientation and position based on the anatomical landmark. Most designs are highly specific and allow only one different size between the femoral or tibial component for total knee replacement $\left(^{5}\right)$. A mismatch between the components will leads to loss of optimal conformity and abnormal load-bearing patterns. Nowadays, many companies advocate the use of templates to enable the surgeon to decide on the appropriate implant size preoperatively. The potential difficulty of accurately determining the magnification of the radiograph is one of the problems in analogue preoperative planning of total knee arthroplasty $\left({ }^{6}\right)$. Besides, the use of templates with standard magnifications does not always allow for accurate correction of the magnification factor $\left({ }^{7}\right)$.

The previous study conducted by Heylen et al., (2016) in 332 patients underwent for TKA surgeries concerning tibial and femoral components size mismatch with no mismatch and Oxford Knee Score (OKS) between the templating, and the actual implant had shown there is no statistically significant difference in OKS between all groups $\left({ }^{8}\right)$. In addition, Miller and Purtil stated that all model predictions were within a \pm 1 size of the TKA templating was acceptable compared with actual components implanted $\left({ }^{9}\right)$. Meanwhile, Trickett et al. claimed that the accuracy of the templated size of the femoral component was $48 \%$. When including for a difference of \pm 1 size, the accuracy of templated size and implant was correct in $98 \%$. The tibial templating also predicted the size of the implant was 55\%, and in all 100\%, including a difference of \pm 1 size during templating $\left({ }^{10}\right)$. 
Digital radiographs are replacing a conventional radiograph to a growing faster. This technology allows the orthopaedic surgeon to plan the procedure on-screen using specialised software. The application of this invention enables the surgeon to correct the magnification factor with more accuracy and reliability $\left({ }^{6,10-12}\right)$. The study conducted by Petretta et al., showed that the acetate templating is similar or better accuracy than digital templating in terms of predicting the sizes to measure the acetabular and femoral components implanted in 52 patients who underwent total hip arthroplasty $\left({ }^{7}\right)$. This study also can be used as a benchmark to improve the accuracy of templating and actual implant in TKA.

However, several drawbacks related to digital templating towards measuring the accuracy of the templating and implant in both components. This technology of advancement is new in Asia, particularly in Malaysia. Millions of dollars are needed to be spender by Malaysia's government to emphasise the used of this digital templating and its maintenance to be implemented in the entire of Malaysia. The semi-automatic digital templating for the knee was invented in National University of Malaysia in 2009 that called OrthoKnee ${ }^{\mathrm{TM}}\left({ }^{13}\right)$. Besides, not all surgeons are familiarised with this digital templating.

Moreover, much skill, advance technique and a better understanding of this technology are needed by the surgeons before used this facility during preoperative planning in TKA $\left({ }^{14}\right)$. Furthermore, times are constraints of much training for better understanding is another factor for consideration to employing this advancement in TKA. To response with the above factors, this study was conducted to evaluate the accuracy of manual templating in tibia and femur for preoperative planning and compare it after implanted in both components in TKA cases $\left({ }^{12}\right)$.

\section{MATERIALS AND METHOD}

This is a prospective study conducted in the Orthopaedic Department, Hospital Tengku Ampuan Afzan (HTAA), Kuantan, Pahang. Patients who planned for the total knee replacement were selected. A total number of 100 cases were chosen. Inclusion criteria include all patients underwent for total knee arthroplasty. The exclusion criteria include fractures, revision joint replacement and improper radiograph magnification.

Preoperative templating in Total Knee Replacement requires standardised radiographs with consistent magnification (120\%). Anteroposterior (AP) and lateral view of the standing knee were taken with magnification markers in situ. The radiograph AP and lateral view were taken $100 \mathrm{~cm}$ from the radiographic plate. Actual size plain radiograph (anteroposterior view and lateral) on standing were taken according to the protocol. The magnification markers were used in all cases to obtain actual size equivalent to $120 \%$ magnification. Manual template device designed by Zimmer ${ }^{\circledR}$ was used in this study, and the magnification of the template device is 
$120 \%$ comparable to $\mathrm{x}$-ray magnification. Pre-templating of the plain radiograph using manual (conventional) template device was used to determine the preoperative implant size.

\section{Methodology of Procedure}

On AP film, the mechanical axis and anatomical axis of the femur and tibia were drawn. The mechanical axis of femur is from the centre of the hip to centre of the knee where the anatomical axis follows the femur bone itself. As for tibia, the mechanical axis is similar to the anatomical axis. The degree of correction was measured for the valgus or varus knee. Drawing lines at the tibial plateau and femoral condyle were performed to determine the joint line level. The tibial cut was planned from the worst articular surface but not lower than the head of the fibula. The aimed of the joint line was transacted 90 degrees to the mechanical axis of the tibia. The osteophytes and degree of correction were identified. A perpendicular line was drawn, and the level of the bone cut was marked. Subsequently, templates with varied sizes were overlaid on the acetate radiograph to obtain proper matching size for tibia component. The joint line was drawn from the femoral condyle, and it must be transacted 5-7 degrees varus as compared to the anatomical axis of the femur. The mechanical axis was maintained from the centre of the hip through the centre of the knee and the centre of the ankle. The line was drawn perpendicular at 90 degrees to the mechanical axis of the femur. The template then was overlaid to the acetate radiograph in order to obtain the matching size. Size of implant used on the day of operation was recorded. All demographic data includes name, age, gender, height, body weight, the preoperative templating and actual size for each patient were taken.

The results were graded 0 for a match, one for mismatch one size different either bigger or smaller by one size, 2 for mismatch 2 sizes and 3 for mismatch 3 sizes. From this grading, the accuracy of the template could be evaluated as a percentage. The percentage of the tibial and femoral component had been calculated.

\section{Statistical Analysis}

Statistical analyses were performed using Student's $t$-test and the analysis of variance (ANOVA) test to compare the accuracy of preoperative templating with the actual implanted sizes between the tibia and femoral components. Using a $95 \%$ confidence interval (CI), a $P$ value less than 0.05 was considered significant.

\section{RESULTS AND DISCUSSION}

A total of one hundred cases of knee replacement were templated in both tibial and femoral components. The implanted size data were collected during the operation.

\section{Patient Demographic}

Malay population has the highest percentage of patients undergoing total knee replacement, which is $48 \%$, followed by Chinese population estimated around $39 \%$ of the population and 
the Indian population is estimated at around $11 \%$. Other ethnic groups were estimated to take around $2 \%$ of the population. In term of patient gender, the female $(\mathrm{N}=75)$ was dominant from the male $(\mathrm{N}=25)$. The distribution of the age of the patients who underwent TKR in this study had been shown in Figure 1. The peak age distribution in this study is ranging from 55 to 65 years of age. The histogram also revealed that the youngest patient that underwent for TKR is at age 38 years old, and the oldest is 82 years old.

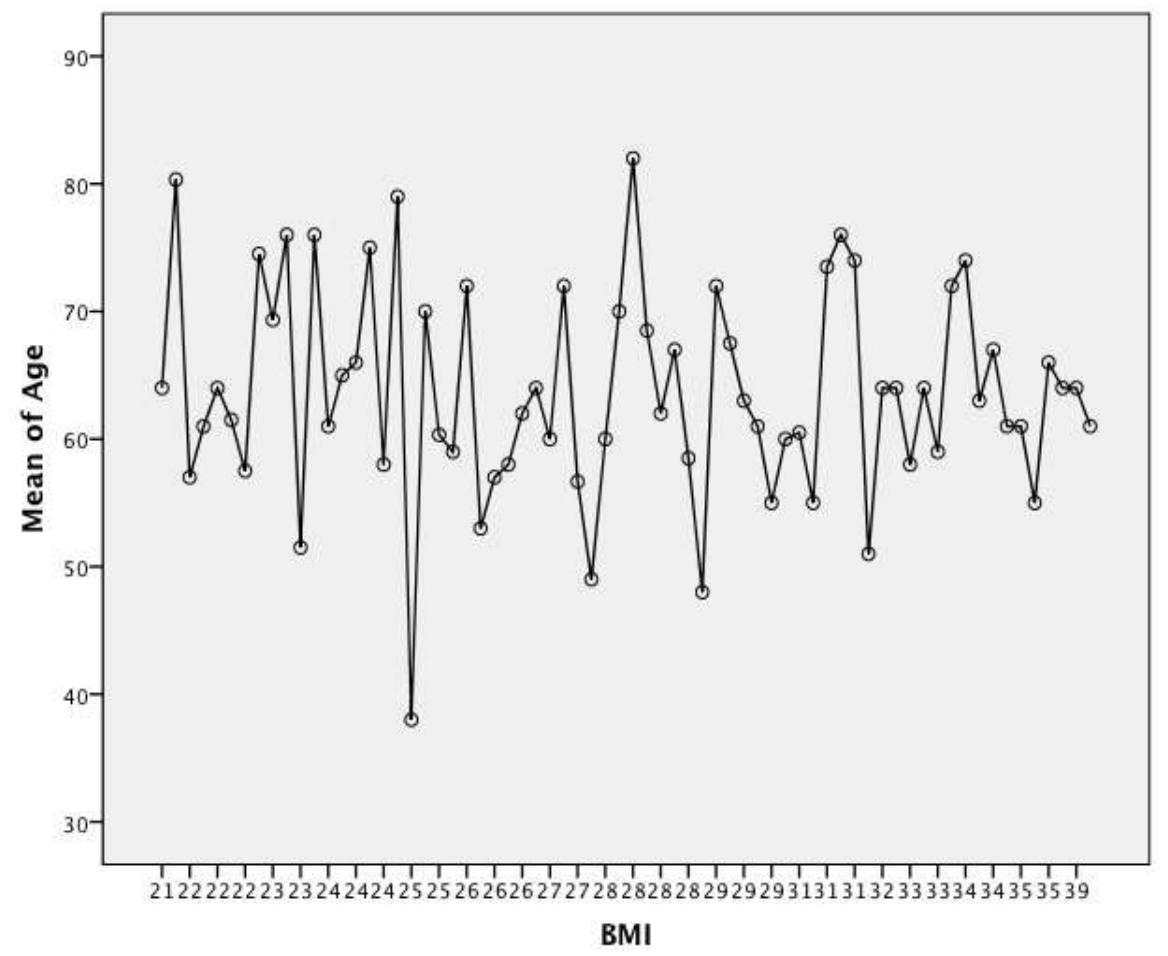

Figure 1: The distribution of the age of the patients who underwent TKR in this study.

\section{Tibial and Femur Component}

The were no significant differences in both tibial $(\mathrm{N}=100)$ and femur $(\mathrm{N}=100)$ component were found in the accuracy of templating and actual implant size in the TKA. The tibial templated $\mathrm{M}=14.29(\mathrm{SD}=18.264)$ showed that no significant difference $(\mathrm{T}=0.695, \mathrm{P}=0.513)$ in all sizes in tibia templating was noted compared to actual implant $\mathrm{M}=11.43(\mathrm{SD}=13.685)$ had been used. By comparison, the femur templating $\mathrm{M}=16.67$ (SD:18.83) also showed there was no significant difference $(\mathrm{T}=0.000, \mathrm{P}=1.000)$ compare with the actual implant in all sizes, as shown in Table 1.

Table 1: The accuracy of the template as compared to the actual size for the tibial component

\begin{tabular}{lllllll}
\hline Group & Size & N & Mean & SD. & $\boldsymbol{t}$ value & $\boldsymbol{p}$. value \\
\hline Templated Tibia & 1 & 1 & 14.29 & 18.264 & 0.695 & 0.513 \\
& 2 & 28 & & & & \\
& 3 & 48 & & & & \\
& 4 & 18 & & & & \\
\hline & 5 & 4 & & & & \\
\hline
\end{tabular}




\begin{tabular}{lllllll}
\hline & 6 & 0 & & & \\
Implanted Tibia & 7 & 1 & & & \\
& 1 & 2 & 11.43 & 13.685 & \\
& 2 & 24 & & & \\
& 3 & 38 & & & \\
& 4 & 20 & & & \\
Templated Femur & 5 & 13 & & & \\
& 6 & 2 & & & & \\
& 7 & 1 & & & \\
& B & 3 & 16.67 & 18.833 & 0.000 & 1.000 \\
& C & 31 & & & & \\
& D & 47 & & & \\
& E & 16 & & & \\
& F & 2 & & & \\
& G & 1 & & & \\
Implanted Femur & B & 4 & 16.67 & 16.777 & \\
& C & 25 & & & \\
& D & 43 & & & \\
& E & 24 & & & \\
& F & 2 & & & \\
& G & 2 & & & \\
\hline
\end{tabular}

In all cases, most of the surgeon have the tendency to choose a smaller size during templating as compared to actual implanted size both in femoral and tibial components. In this study, the mismatch condition can occur at all sizes on the tibia. Therefore, the mismatch of implant sizes can occur in this procedure. As Showed in Figure 3, The most popular acetate templating in the tibial component used is size $3(T=48)$ the actual implant $(A=38)$ the different $(D=10)$, followed by size $2(N=24)$, size $2(T=28, A=24, D=4)$, size $4(T=18, A=20, D=2)$, size $5(\mathrm{~T}=4, \mathrm{~A}=13, \mathrm{D}=9)$, size $6(\mathrm{~T}=0, \mathrm{~A}=2, \mathrm{D}=2)$ and the lowest mismatch is size $1(\mathrm{~T}=$ $1, \mathrm{~A}=2, \mathrm{D}=1)$ and size 7 has no mismatch $(\mathrm{T}=1, \mathrm{~A}=1)$.

During implantation, the similar pattern showed the mismatch of size in the determination of implant during the templating procedure. Figure 4 demonstrated that the most popular acetate templating in tibial component used is size $D(T=47, A=43, D=5)$, followed by size $C(T=31$, $A=25, D=6)$, size $E(T=16, A=24, D=8)$, size $B(T=3, A=4, D=1)$, and the lowest mismatch is size $G(T=1, A=2, D=1)$ and size $F$ has no mismatch $(T=2, A=2)$.

The accuracy of the template as compared to the actual size is $48 \%$ for tibial component, and when it includes one different size, it is accounted for 95\% accuracy as illustrated in Table 2. There is no difference between more than 2 sizes. The accuracy and reliability of manual templating had been used in this study. It shows that $48 \%$ of the tibial component being templated is equal to the size of the implant being used. When the difference of one size (above or below) is included, the accuracy of manual templating reached 95\% (Figure 2). There are no cases of templating given result in more than 3 size differences. 


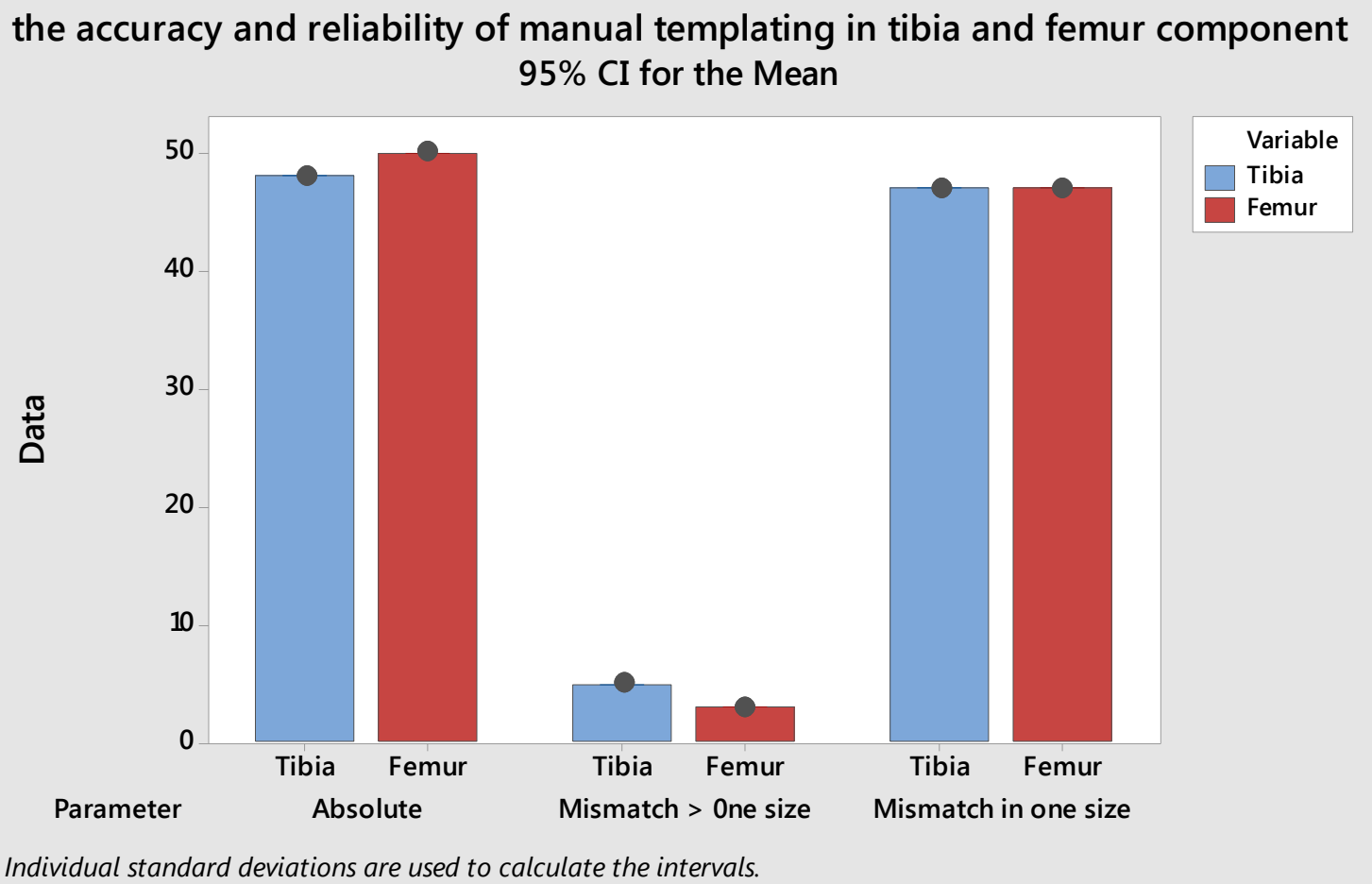

Figure 2: The bar chart showed the accuracy and reliability of manual templating in a different size in the accuracy of the tibial and femur component.

Table 2: The accuracy of templating and implanted in both tibia and femur in TKA

\begin{tabular}{lllll}
\hline Tibia & \multicolumn{5}{c}{$\begin{array}{l}\text { Accuracy + within } \\
\text { one size mismatch } \\
\text { Size Different }\end{array}$} & $\begin{array}{l}\text { N } \\
\text { Accurately template }\end{array}$ & $\begin{array}{l}\text { SD } \\
\text { Within one size mismatch }\end{array}$ & 47 & 0 & 95 & $\begin{array}{l}\text { More than one size } \\
\text { mismatch (per cent) }\end{array}$ \\
More than one size mismatch & 5 & 0 & - & - \\
Femur & & & & - \\
Accurately template & 47 & & 97 & - \\
Within one size mismatch & 50 & & & - \\
More than one size mismatch & 3 & & & 3 \\
\hline
\end{tabular}

The bar chart (Figure 2) shows the accuracy and reliability of manual templating in this study. It shows that $48 \%$ of the tibial component being templated is equal to the size of the implant being used. When the difference of one size (above or below) was included, the accuracy of manual templating reached $95 \%$. There are no cases of templating given a result of more than 3 size differences. While, as for the percentage of accuracy of templating and actual implant for femoral components were shown in Figure 3. The accuracy of templating for femoral components is $47 \%$. When one size difference is included, the accuracy of up to $97 \%$ has been demonstrated. There is only $3 \%$ of templating size difference of 2 sizes, while no differences of 3 or more size are recorded. 


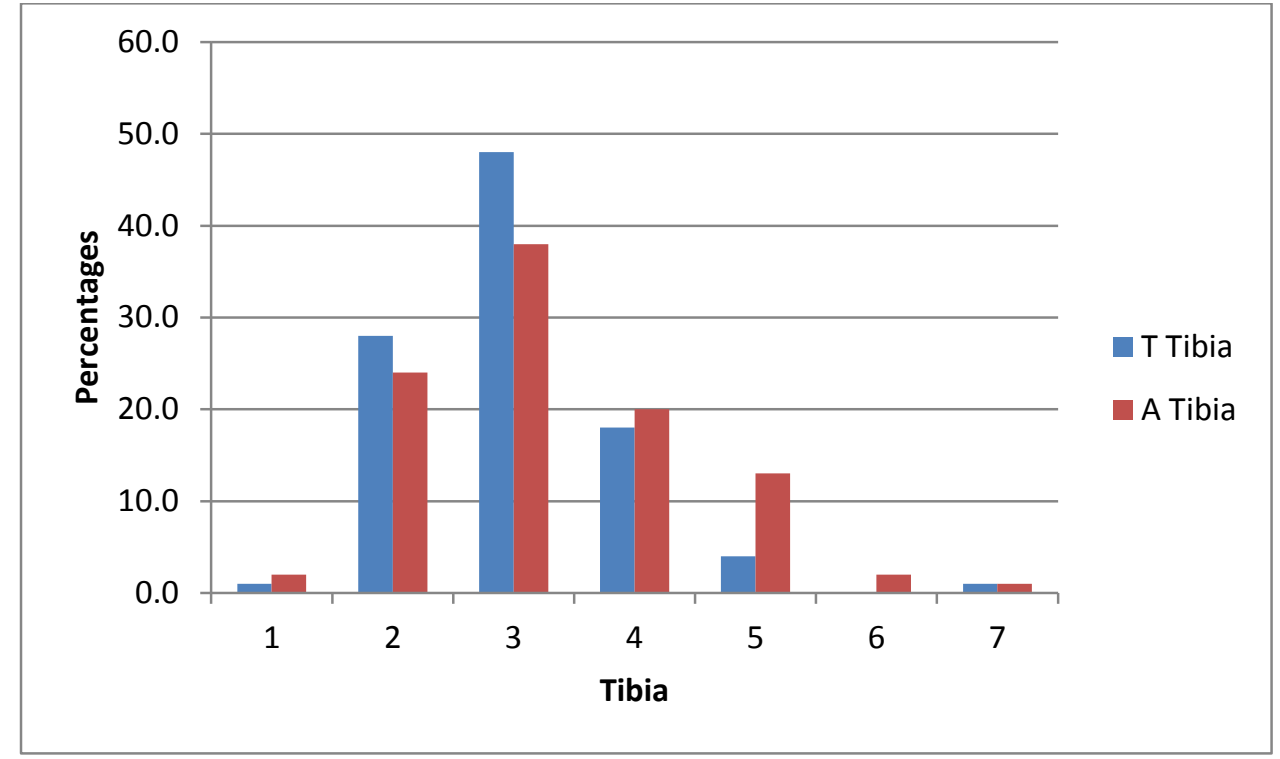

Figure 3: Histograms depicting the size difference between the templated and implanted of the tibial component, which shows the accuracy difference between the acetate templating and tibial implant.

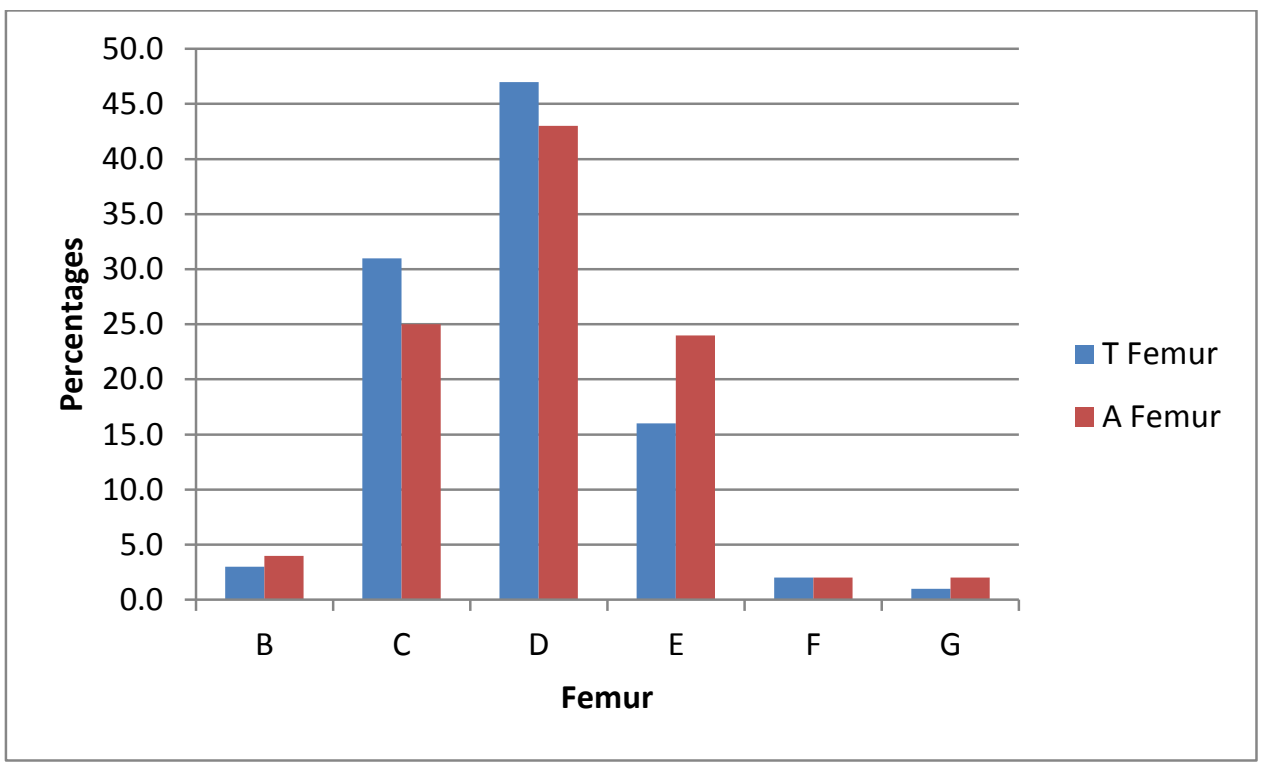

Figure 4: Histograms depicting the difference size between the templated and implanted of the femoral component, which shows the accuracy difference between the acetate templating and femoral implant.

TKA is one of the most successful procedures in the orthopaedic field. The significant goals of arthroplasty are to relieve pain effectively, restoring range of motion and helps to improve function $\left({ }^{4}\right)$. Due to the rising new technology advancement with the introduction of new implant design makes preoperative planning of total knee arthroplasty is a crucial part of the surgical procedure. This practised is not only allowed the surgeon to think three-dimensionally but also thought them to improve surgical precision, shorten the length of the surgical procedure, and reduce the incidence of complications. This study was purposely conducted to 
access the accuracy of manual templating is reliable with the size for TKA as not all facilities in operation theatre have sophisticated digital templating systems.

A total of 100 cases have been selected in this study. The most common underlying disease that leads to total knee replacement is the primary osteoarthritis, and the youngest patient who underwent TKA was due to severe Rheumatoid Arthritis. The distribution of age undergoing total knee replacement predominantly ranging from 55 to 65 years of age, the youngest being 38 years old and the oldest is 82 years old. The most common underlying disease that leads to total knee replacement is the primary osteoarthritis, and the youngest patient who underwent TKA was due to severe Rheumatoid Arthritis. In term of gender distribution, $75 \%$ of the cases are female patients, and $48 \%$ of the cases are dominated by the Malay population, followed by Chinese, Indian and others. The percentage is the reflection of a public social demographic in Kuantan, Pahang where Malay population is dominant. Other races include Orang Asli, and in one case there is a Caucasian, which required more significant implant size $\mathrm{H}$ for femur and 7 for the tibial tray. The incidence of total knee replacement in this study is higher on the right knee as compared to the left knee at $55 \%$ and $45 \%$ respectively.

Based on the result being shown previously, we found that the accuracy of tibial components in total knee replacement is $48 \%$ using $\mathrm{Zimmer}^{\circledR}$ Template System. When the accuracy of the templating is encountered within one size difference, the accuracy is $95 \%$. Only $5 \%$ of cases are templated at the difference of two sizes. The accuracy of femoral component templating using Zimmer ${ }^{\circledR}$ template design is $47 \%$. Another $50 \%$ of cases templated within one size difference (accuracy within one size 97\%). Size D is the most used size in TKA with almost half of the case followed by size C, and E. Data from this study showed only three per cent of cases templated in two size differences. None of the cases templated more than 2 sizes.

The tibial and femoral components size predictions are only correct in about half of patients who underwent TKA. However, when allowing a discrepancy of \pm 1 size, the accuracy increased to more than $95 \%\left({ }^{10}\right)$. As compared to others, Howcroft comparing 3 prosthetic designs in the United Kingdom (PFX, Kinemax and Scorpio) showed quite a wide range of accuracy for tibial and femoral component and Scorpio became the most accurate ranging from $72-75 \%$ for tibial templates. However, the PFC template design showed inferior results, where the range of accuracy was between 53-58\%. They concluded that the difference of magnification of radiograph in each design (115\% vs $110 \%)$ contributed to the full range of accuracy of templating $\left({ }^{5}\right)$. Larry W. Carter et al. found that the templated size corresponded to the actual femoral implant used in approximately $50 \%$ of cases. When femoral prostheses within one size above or below the templated size were included, the accuracy of preoperative templating rose to $88-95 \%\left({ }^{15}\right)$. When implants within two sizes of the templated size were 
included; the accuracy approached 100\%. A study conducted by Heal and Blewitt (Year), assessed the accuracy of the femoral component using the Kinemax system in AP and Lateral view. They found that in AP view, the accuracy of templating was 53\% while in Lateral view, it was $63 \%\left({ }^{16}\right)$.

From this study, we have observed that there are several reasons for the inaccuracy of the manual templates, which includes the interobserver and intraobserver reliability, the rotation of the radiograph and fixed flexion deformity. Howcroft et al. had studied the interobserver and intraobserver reliability, and he found a significant result of templating in two different groups $\left({ }^{5}\right)$. However, in the study conducted by Bothra et al. showed the comparing result of the accuracy and reliability of templating measurement between senior consultants and junior surgeons were found that no obvious association between K-coefficient and seniority $\left({ }^{17}\right)$.

The second factor, the rotation of the radiographs leading to the error of measurement and inaccurate size had been selected. In this study, the recent before the operation and best radiographs were chosen before templated. Although acetate templating was found accurate and reliable during TKA, the use of digital radiographs with the digital templating has recently grown is crucial due to advances in reducing errors associated with manipulating films and templates $\left({ }^{3,13,14}\right)$.

Third, the fundamental error in fixed flexion deformity will increase the magnification where the distance knee from the x-ray plate is reduced. The error induced by flexion deformity could be eliminated by increasing the distance from the X-ray source to the knee in correlation to increasing flexion deformity and reducing the magnification. According to Ballermans et al., the alignment and joint-space aberration need to be increased the magnification from X-ray beam parallax, causing the templating inaccurate in fixed flexion deformity $\operatorname{cases}\left({ }^{4,18}\right)$.

\section{CONCLUSION}

This study could be giving more insight the need for accurate measurements of acetate templating and reliability of implant in tibial and femur components during the preoperative procedure for TKA cases. It will also not only help the surgeon in the selection of appropriate implant size, but also correct accuracy measurement to minimise the mismatch during implantation. Further detailed morphometric analysis would be needed to help in selecting the implants as required.

\section{ACKNOWLEDGEMENT}

We thank our colleagues from the Department of Orthopedics, Tengku Ampuan Afzan Hospital, Kuantan who provided insight and expertise that greatly assisted the research, resulting in all of the interpretations and conclusions of this paper successfully published. 


\section{REFERENCES}

1. Jain J. Knee prosthesis sizes in Indian patients undergoing total knee replacement. Int Surg J. 2015;2(3):348-351. doi:10.18203/2349-2902.isj20150497

2. Rahul V, Andrew JK, David WS. US Patent Application for method and computer program product for preplanning orthopaedic surgery Patent Application (Application \#20180085165 issued March 29, 2018) - Justia Patents Search. Justia Patents. https://patents.justia.com/patent/20180085165. Published in 2018. Accessed September 12, 2019.

3. Erdogan AO, Gokay NS, Gokce A. Preoperative Planning of Total Knee Replacement. Arthroplast - Updat. 2013;(June). doi:10.5772/55023

4. Tanzer M, Makhdom AM. Preoperative Planning in Primary Total Knee Arthroplasty. J Am Acad Orthop Surg. 2016;24(4):220-230. doi:10.5435/JAAOS-D-14-00332

5. Howcroft DWJ, Fehily MJ, Peck C, Fox A, Dillon B, Johnson DS. The role of preoperative templating in total knee arthroplasty: Comparison of three prostheses. Knee. 2006;13(6):427-429. doi:10.1016/j.knee.2006.05.002

6. The B, Verdonschot N, van Horn JR, van Ooijen PMA, Diercks RL. Digital Versus Analogue Preoperative Planning of Total Hip Arthroplasties. A Randomized Clinical Trial of 210 Total Hip Arthroplasties. J Arthroplasty. 2007;22(6):866-870. doi:10.1016/j.arth.2006.07.013

7. Petretta R, Strelzow J, Ohly NE, Misur P, Masri BA. Acetate Templating on Digital Images Is More Accurate Than Computer-based Templating for Total Hip Arthroplasty. Clin Orthop Relat Res. 2015;473(12):3752-3759. doi:10.1007/s11999015-4321-y

8. Heylen S, Foubert K, Van Haver A, Nicolai P. Effect of femoro-tibial component size mismatch on outcome in primary total knee replacement. Knee. 2016;23(3):532-534. doi:10.1016/J.KNEE.2016.03.003

9. Miller AG, Purtill JJ. Total Knee Arthroplasty Component Templating: A Predictive Model. J Arthroplasty. 2012;27(9):1707-1709. doi:10.1016/J.ARTH.2012.03.055

10. Trickett RW, Hodgson P, Forster MC, Robertson A. The reliability and accuracy of digital templating in total knee replacement. J Bone Joint Surg Br. 2009;91-B(7):903906. doi:10.1302/0301-620X.91B7.21476

11. Iorio R, Siegel J, Specht LM, Tilzey JF, Hartman A, Healy WL. A Comparison of Acetate vs Digital Templating for Preoperative Planning of Total Hip Arthroplasty. J Arthroplasty. 2009;24(2):175-179. doi:10.1016/j.arth.2007.11.019 
12. Peek AC, Bloch B, Auld J. How useful is templating for total knee replacement component sizing? Knee. 2012;19(4):266-269. doi:10.1016/j.knee.2011.03.010

13. Yusof SF, Sulaiman R, Thian Seng L, et al. Development of total knee replacement digital templating software. Lect Notes Comput Sci (including Subser Lect Notes Artif Intell Lect Notes Bioinformatics). 2009;5857 LNCS:180-190. doi:10.1007/978-3-64205036-7_18

14. Kniesel B, Konstantinidis L, Hirschmüller A, Südkamp N, Helwig P. Digital templating in total knee and hip replacement: an analysis of planning accuracy. Int Orthop. 2014;38(4):733-739. doi:10.1007/s00264-013-2157-1

15. Carter LW, Stovall DO, Young TR. Determination of accuracy of preoperative templating of noncemented femoral prostheses. J Arthroplasty. 1995;10(4):507-513. doi:10.1016/s0883-5403(05)80153-6

16. Heal J, Blewitt N. Kinemax total knee arthroplasty: Trial by template. J Arthroplasty. 2002;17(1):90-94. doi:10.1054/arth.2002.27258

17. Bothra V, Lemon G, Lang D, Smith DM, Ali AM. Reliability of templating in estimating the size of uni-condylar knee arthroplasty. J Arthroplasty. 2003;18(6):780783. doi:10.1016/s0883-5403(03)00112-8

18. Bellemans J, Vandenneucker H, Victor J, Vanlauwe J. Flexion contracture in total knee $\begin{array}{lllll}\text { arthroplasty. Clin } & \text { Orthop Relat Res. 2006;(452):78-82. }\end{array}$ doi:10.1097/01.blo.0000238791.36725.c5

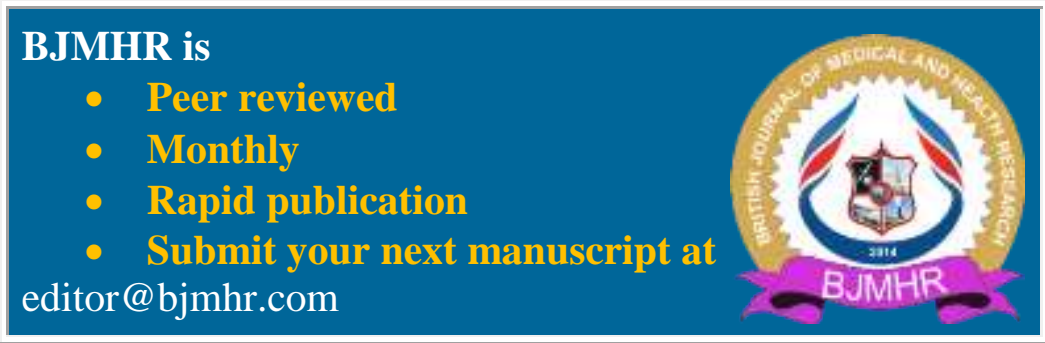

